\title{
LÓGICA INFORMAL: UMA VISÃO GERAL
}

\author{
Ralph H. Johnson ${ }^{\mathrm{ii}}$ \\ J. Anthony Blairiii
}

Resumo: Neste artigo, primeiramente, explicamos o que entendemos por lógica informal, discutimos equívocos e diferenciamos nossa concepção das demais; em segundo lugar, catalogamos brevemente pesquisas recentes sobre lógica informal, divididas em quatorze tópicos; em terceiro, sugerimos quatro áreas gerais de problemas e questões para futuras pesquisas; em quarto, descrevemos os atuais recursos acadêmicos para a lógica informal; em quinto, discutimos três implicações da lógica informal para a filosofia, em particular, e ressaltamos consequências práticas de modo mais geral.

Palavras-chave: Teoria da Argumentação. Lógica Formal. Esquemas Argumentativos. Recursos da Lógica Informal.

Abstract: In this overview article, we first explain what we take informal logic to be, discussing misconceptions and distinguishing our conception of it from competing ones; second, we briefly catalogue recent informal logic research, under 14 headings; third, we suggest four broad areas of problems and questions for future research; fourth, we describe current scholarly resources for informal logic; fifth, we discuss three implications of informal logic for philosophy in particular, and take note of practical consequences of a more general sort.

Keywords: Argumentation theory. Formal logic. Argument schemes. Informal logic resources.

\footnotetext{
'A Revista EID\&A agradece vivamente a J. Anthony Blair, Coeditor da Revista Informal Logic, pela autorização da publicação desta tradução do artigo originalmente publicado como: BLAIR, J. A.; JOHNSON, R. H. Informal Logic: an overview. Informal Logic, Windsor, v. 20, n. 2, p. 93-107, 2000.

ii Professor emérito da University of Windsor, Canadá. E-mail: johnsoa@uwindsor.ca

iii Professor emérito da University of Windsor, Canadá. E-mail: tblair@uwindsor.ca
} 
EID\&A - Revista Eletrônica de Estudos Integrados em Discurso e Argumentação, Ilhéus, n. 14, jul/dez.2017.

\section{Introdução}

Os assuntos abordados neste artigo estão contidos nas questões propostas ao simpósio sobre lógica informal e filosofia durante o Congresso Mundial de Filosofia ${ }^{1}$ em Boston, em agosto de 1998:

- Qual a relevância filosófica da lógica informal?

- Quais são as aplicações/implicações da lógica informal para as outras áreas da filosofia?

Não pretendemos aqui responder a essas questões detalhadamente, contudo nossas visões tornar-se-ão evidentes ao fim de nossa explanação, a qual se dará da seguinte forma: começaremos com uma breve exposição sobre a natureza da lógica informal, sobre o que ela é e o que não é. Delinearemos, então, os problemas e as questões que a lógica informal aborda, seguido de um breve relato dos recentes desenvolvimentos neste campo, dos recursos disponíveis, e, finalmente, de nossa visão sobre as implicações da lógica informal para a filosofia.

\section{Lógica informal: o que é?}

A. Rumo a uma definição. Muitos notaram a forte presença canadense nas pesquisas do campo da lógica informal. Talvez coincidentemente, tanto canadenses quanto lógicos informais parecem estar envolvidos em um questionamento incessante sobre sua identidade. O que é ser canadense, diferentemente de ser americano, britânico, francês e assim por diante? Lógicos informais se perguntam: o que é a lógica informal, diante da lógica dedutiva formal, da epistemologia e do pensamento crítico? Walton e Brinton (1997, p. 9), em uma antologia recente sobre a história da lógica informal, afirmam que:

A lógica informal ainda não se unificou como uma disciplina definida e organizada em torno de técnicas sistemáticas bem definidas consolidadas, dotadas de uma estrutura definitiva e passíveis de serem categoricamente aplicadas por seus praticantes.

Essa observação é significativa, ainda que incômoda, na medida em que, embora permaneçam equívocos sobre o que a lógica informal aborda, seus principais teóricos (entre os quais incluímos Walton, Govier e nós mesmos)

\footnotetext{
1 Acrescentamos as referências de alguns dos trabalhos que apareceram posteriormente ao Congresso Mundial.
} 
EID\&A - Revista Eletrônica de Estudos Integrados em Discurso e Argumentação, Ilhéus, n. 14, jul/dez.2017.

tendem a concordar que a lógica informal trata do estudo das normas dos argumentos (em vez de lidar com as normas que regulam inferências ou implicações). Propomos, novamente (ver JOHNSON; BLAIR, 1987, p. 148), a seguinte maneira de interpretar a lógica informal, que corrobora o que ambos, Govier (1987) e Walton (1989), propõem:

A lógica informal designa o ramo da lógica cuja função é desenvolver parâmetros, critérios e procedimentos não-formais para a análise, interpretação, avaliação, crítica e construção da argumentação no discurso cotidiano.

"Não-formal" é um termo emprestado da distinção empregada por Barth-Krabb (1982) acerca dos três sentidos de "forma". Essa lógica é nãoformal nas acepções seguintes: ela não se vale da ferramenta analítica central da lógica dedutiva formal - a noção de forma lógica -, nem se orienta pela sua principal função avaliativa da lógica dedutiva formal, a validade. Entretanto, isso não significa que essa lógica seja não-formal no sentido de que abandona referências a parâmetros, critérios ou procedimentos.

Um problema com essa definição, contudo, é que ela limita a lógica informal ao discurso cotidiano, uma restrição que nos parece agora desnecessária e contrafactual. Historicamente, o domínio de interesse da lógica formal tem sido o que pode ser chamado de "argumentação em língua natural", a qual possui dois subdomínios: (a) o discurso cotidiano (discussões de assuntos públicos, como ocorrem em editoriais de jornais) e (b) o que Weinstein (1990) chamou de discurso "estilizado" - modalidade que abrange os estilos de argumentos específicos de um domínio, além das formas de inferência e das epistemologias de disciplinas típicas como, por exemplo, as diferentes ciências. A divisão crucial não é entre discursos cotidianos e os estilizados, mas entre línguas artificiais e naturais. Essas últimas são o ponto focal da lógica informal (diferente do foco da lógica dedutiva formal, que se volta para as línguas artificiais e os sistemas lógicos), qual seja o discurso.

B. Alguns equívocos e concepções concorrentes sobre lógica informal. Apresentaremos, nesta subseção, um inventário de outras respostas à pergunta: então, o que é lógica informal? Algumas dessas respostas são facilmente reconhecidas como erradas por qualquer pessoa minimamente familiarizada com a área nos últimos vinte e cinco anos; outras, contudo, partem de colegas especialistas de quem discordamos. Começaremos com uma resposta que alguns consideram um locus classicus - Ryle (1954) utiliza o rótulo 'lógica informal' para referir-se às implicações de conceitos 
EID\&A - Revista Eletrônica de Estudos Integrados em Discurso e Argumentação, Ilhéus, n. 14, jul/dez.2017.

substantivos (como tempo e prazer), cuja lógica é "informal”, em oposição à lógica de conceitos como conjunção e disjunção, cuja lógica é "formal”. Para Ryle, "lógica informal” parece ser sinônima de "análise filosófica". Trata-se de uma compreensão muito mais ampla do que aquela que será encontrada nos trabalhos dos principais teóricos da área.

Um segundo equívoco sobre a lógica informal é que ela se restringe exclusivamente ao estudo das falácias informais (CARNEY; SHEER 1964, KAHANE, 1971). O estudo em questão constitui, sim, uma parte - mas apenas uma parte - do objeto de investigação da lógica informal.

Um terceiro equívoco acerca da lógica informal é o de que ela consiste em uma lógica formal que prescinde da formalidade. Essa ideia é ilustrada pelo modo com que Copi produziu seu manual, Informal Logic (1986), que consiste simplesmente em excertos de seu livro Introduction to Logic (1986), com os capítulos sobre lógica formal omitidos. Essa visão é incorreta, pois, por um lado, onde o formalismo mostra, a lógica informal emprega (ver WOODS; WALTON, 1982); e, por outro lado, o foco principal da lógica informal é o que o formalismo, nesse sentido, não mostra.

Um ponto de discordância dentro da área é a visão de que a lógica informal teria a tarefa de mediar a lógica formal e o raciocínio em língua natural. Tal ponto de vista pode ser encontrado em Goldman (1986) e Woods (1995). Para nós, nos casos em que implicações dedutivas ocorrem na argumentação em língua natural, elas podem ser formalizadas (às vezes, vantajosamente, outras, não). Implicações que não são acarretamentos, e outros aspectos da análise e da avaliação dos argumentos, não são passíveis de tratamento formal.

Uma segunda visão da qual discordamos (defendida por MCPECK, 1981; SIEGEL, 1988; e WEINSTEIN, 1994) é a de que a lógica informal seja epistemologia aplicada, no sentido de que ela se vale da aplicação de evidências epistemológicas para a avaliação de argumentos. Nós poderíamos até concordar, mas apenas se a noção de epistemologia em questão fosse ampliada de forma a incluir a lógica, a dialética e a retórica.

Por fim, discordamos de Fisher e Scriven (1997, p. 76), que asseveram que a lógica informal é "a disciplina que estuda a prática do pensamento crítico e lhe fornece sua espinha dorsal intelectual". Considerando, por um lado, que o objeto de estudo da lógica informal são os argumentos e a argumentação, e, por outro, que o pensamento crítico, na visão de Fisher e 
EID\&A - Revista Eletrônica de Estudos Integrados em Discurso e Argumentação, Ilhéus, n. 14, jul/dez.2017.

Scriven (1997, p. 21), é "a hábil e ativa interpretação e avaliação das observações e das interações, da informação e da argumentação”, atribuímos à lógica informal um escopo mais estreito do que esses autores.

\section{Pesquisas recentes}

Nesta seção, voltamo-nos para algumas das áreas de pesquisa mais recentes no campo da lógica informal.

1. A história da lógica informal. Um desenvolvimento importante foi a emergência de trabalhos sobre a história da lógica informal. Por vezes é dito que pouquíssima coisa foi feita na área desde o período de publicação da obra de Aristóteles e a publicação de Fallacies (HAMBLIN, 1970) e Logic and Contemporary Rhetoric (KAHANE, 1971). No entanto, a antologia de Hansen e Pinto (1995) Fallacies, Classical and Contemporary Readings inclui diversos estudos de tratamentos históricos sobre as falácias informais. Além disso, a Historical Foundations of Informal Logic, de Walton e Brinton (1997), com capítulos sobre Isaac Watts, Whately, Bentham, Mill e Kant, mostra quantos trabalhos relevantes foram realizados nesse intervalo de mais ou menos dois milênios.

2. Argumentação como diálogo. Uma segunda área de pesquisa, levada a cabo por Walton (1996) e Walton e Krabbe (1995), tem sido o modelamento de argumentos como diálogos de diferentes tipos. Seus trabalhos podem ser vistos como uma elaboração da teoria pragmadialética, com a qual mantêm laços estreitos. É também possível ver a influência da Pragmadialética na teoria de falácias dos autores. Mais recentemente, algumas limitações do modelo dialógico têm sido sugeridas (TINDALE, 1996; BLAIR, 1998).

3. Ampliando o conceito de argumento. Diversos pesquisadores têm sugerido a revisão do conceito de argumento em outras direções. Gilbert (1997) defende que os argumentos não são exclusivamente verbais, mas também emocionais, viscerais e "kiscerais"،. Groarke (1996), Birdsell e Groarke (1996), além de Blair (1996), também entendem que comunicações visuais, tais como obras-de-arte, sejam argumentos. Mais recentemente, Tindale (1999) defendeu que se considere a perspectiva retórica como básica para a noção de argumento.

\footnotetext{
${ }^{2}$ N.T.: O termo "kisceral”, elaborado por Gilbert, abrange o campo das experiências intuitivas e não sensoriais, o místico, o religioso, o sobrenatural e o extrassensorial.
} 
EID\&A - Revista Eletrônica de Estudos Integrados em Discurso e Argumentação, Ilhéus, n. 14, jul/dez.2017.

4. Razão e argumentação. A relação entre razão e argumentação tem sido explorada na literatura recente em dois níveis. No nível micro, elabora-se a distinção e discute-se a relação entre raciocínio e argumentação (PINTO, 1995; BLAIR, 1999). No nível macro, explora-se a conexão entre a argumentação e a racionalidade - tanto o lugar da argumentação na racionalidade, quanto às coerções que a mesma exerce sobre a argumentação (ver WALTON, 1990; FINOCCHIARO, 1992; JOHNSON, 1996).

5. Nova teoria da inferência. Ao apresentar fundamentos/dados para sustentar uma alegação, pode-se acreditar que eles impliquem a alegação de maneira dedutiva ou que eles lhe forneçam forte apoio indutivo. Tradicionalmente, essas duas alternativas eram vistas como as únicas formas possíveis de se apoiar racionalmente uma tese. Tomando como base Wellman e Wisdom, Govier (1987) há muito defende que existam outras maneiras pelas quais fundamentos podem apoiar uma alegação. Wellman (1971) usou o termo "condução" em paralelo à dedução e à indução. Rescher (1976) usou o termo "raciocínio plausível" em uma conexão similar. Mais recentemente, Scriven (1987), usando o termo "raciocínio probatório", e Walton (1996b), "raciocínio presumível", argumentaram por um terceiro tipo de apoio evidencial. Esses autores apontam a direção de uma nova teoria da inferência.

6. Esquemas argumentativos. Nos últimos anos, a ideia de que os esquemas argumentativos possam ter a chave para importantes problemas na teoria da argumentação tem emergido na pesquisa de vários acadêmicos. Kienpointner (1992) identificou mais de 80 esquemas argumentativos e classificou-os em uma tipologia organizacional. Walton (1996b) encontra nos esquemas argumentativos padrões de raciocínio presumível e entende diversas falácias como mau uso ou abuso de esquemas argumentativos.

7. Estruturas e diagramas. Na literatura, foi dedicada considerável atenção à estrutura dos argumentos dentro da argumentação e aos seus modelos de diagramação. Freeman (1991) forneceu um modelo inspirado em Toulmin e em uma noção dialética do argumento; Snoeck Henkemans (1992) ofereceu uma análise embasada na abordagem Pragmadialética; já Walton (1996a) desenvolveu sua própria análise pragmática, fundamentada no diálogo.

8. Teoria da falácia. O estudo das falácias está fortemente associado à lógica informal. Diversos desenvolvimentos importantes ocorreram a partir da clássica série de artigos de Woods e Walton sobre falácias específicas 
publicados na década de 1970 e no início dos anos 1980, reunidos na obra Fallacies: Selected Papers, 1972-1982, de 1989. Em primeiro lugar, a excelente coleção de artigos e pesquisas editada por Hansen e Pinto (1995) apontada acima, Fallacies: Classical and Contemporary Readings (1995). Em segundo lugar, Walton (1995) propôs um tipo de classificação ou hierarquia de erros. Em terceiro lugar, houve uma mudança na área, que passou a enxergar a falácia como um certo tipo de erro procedimental ou estratégico (WALTON; KRABBE, 1995; VAN EEMEREN; GROOTENDORST, 1992; HINTIKKA, 1987). Em quarto lugar, devemos mencionar a contínua pesquisa de Walton sobre as falácias. $\mathrm{Na}$ última década, Walton vem produzindo uma série de extensos estudos sobre falácias específicas: até o momento, ele publicou monografias sobre a petição de princípios (1991), apelo à emoção (1992a), "falácia da ladeira escorregadia (slippery slope)" (1992b), argumento da ignorância (1994) e o argumento ad hominem (1998). Ademais, além de numerosos artigos sobre falácias específicas, ele publicou duas monografias acerca da teoria da falácia e sobre falácias em geral (WALTON, 1987, 1995).

9. Adequação de premissas. Ao contrário da moderna tradição lógica de ignorar questões sobre adequação doxástica, epistêmica ou dialética das premissas de argumentos, o foco da lógica informal na avaliação de argumentos levou seus seguidores a debruçarem-se sobre critérios de adequação de premissas. Há um interesse razoavelmente disseminado sobre a tríade relevante-suficiente-aceitável, proposta primeiramente por Johnson e Blair (1977). Entre os autores que abraçaram essa perspectiva (por vezes, com terminologia ligeiramente diferente) estão: Govier (1985), que troca "suficiente" por “adequado", Darner (1987), Freeman (1988), Little, Groarke e Tindale (1989), Barry (1992) e Seech (1992). Johnson e Blair (1994), Pinto e Blair (1993) e Freeman (1992) defenderam uma normatização acerca da aceitabilidade de premissas, uma modificação do modelo de Van Eemeren e Grootendorst (1984). Mais recentemente, Blair (1995) assumiu uma posição relativista, enquanto Johnson (1998) insistiu na verdade como um dos critérios de adequação de premissas. Inúmeros artigos em lógica informal acerca da noção de relevância foram publicados na última década (ver, por exemplo, os artigos de Tindale, Woods, Blair, Freeman e Hitchcock na edição especial de 1990 da revista Argumentation sobre o tema). A noção de suficiência tem recebido menos atenção (ver, contudo, BLAIR, 1991). 
10. Desenvolvimentos na teoria da crítica argumentativa. Walton (1995) defende que a crítica argumentativa não é consistente: algumas teorias são mais sérias do que outras e devemos diferenciar, por exemplo, entre execução fraca e um mau uso ou abuso. Ele também apresenta uma possível tipologia das falácias, distinguindo entre paralogismos e sofismas. Johnson (1996) defende uma distinção entre a avaliação e a crítica argumentativa, e advoga que é preciso desenvolver princípios de crítica, tais quais: o princípio da vulnerabilidade, o princípio da discriminação e o princípio da neutralidade lógica. Os dois últimos foram incorporados à Logical Self-Defense, de Johnson e Blair (1994), mas discussões subsequentes são necessárias para refinar sua formulação e sua justificação, bem como para verificar se há outros princípios.

11. A investigação do papel social da argumentação. No desenvolvimento de uma teoria da crítica da argumentação, Johnson (1996) examinou o papel da argumentação como prática social e propôs normas para as obrigações sociais dos oradores no que se refere tanto ao uso apropriado da prática quanto à manutenção de seu vigor. Govier (1987) e Walton (1980) também chamam atenção para o papel da argumentação na vida da sociedade.

12. O impacto da teoria feminista. O exame crítico feminista das metáforas empregadas na prática e na teorização sobre a argumentação levou à reavaliação dos mesmos (ver AYIM, 1998). A preocupação geral acerca do privilégio do racional e a marginalização do emocional levou a um debate entre lógicos informais sobre ampliar a concepção de argumentação e sobre limitar o seu papel social (ver ORR, 1989; NYE, 1990; GOVIER, 1993; MENSSEN, 1993, GILBERT, 1994). Tanto Gilbert (1997) quanto Tindale (1999) dedicaram capítulos favoráveis à influência dos insights feministas para a teoria do argumento.

13. Retorno à retórica. É devido aos Acts of Argument, de Tindale (1999), que uma perspectiva retórica se tornou tanto essencial quanto fundamental para uma teoria do argumento. Coalescent Argument, de Gilbert (1997), também propõe uma mudança voltada para uma perspectiva retórica.

14. Argumentação como pragmática. Walton, há algum tempo, vem desenvolvendo uma teoria pragmática da argumentação (1995), e o livro recente de Johnson, Manifest Rationality: A Pragmatic Theory of Argument (2000), apresenta uma teoria argumentativa que, embora divirja em muitos aspectos da proposta de Walton, é igualmente pragmática. 
EID\&A - Revista Eletrônica de Estudos Integrados em Discurso e Argumentação, Ilhéus, n. 14, jul/dez.2017.

\section{Problemas e Questões para pesquisas futuras}

Os recentes desenvolvimentos teóricos listados anteriormente representam um programa de pesquisa em curso para a lógica informal. Nesse sentido, esta seção é constituída por "problemas e questões para pesquisas futuras" que estão estimulando a atual pesquisa no campo. Ainda assim, uma classificação dos problemas pode ser útil. Sob nossa perspectiva, existem quatro áreas de "problematização" - todas elas claramente inter-relacionadas - em torno das quais a pesquisa em teoria da lógica informal pode ser organizada.

1. A teoria do argumento/da argumentação. Como argumentos e argumentação podem ser entendidos? Alguns teóricos, como os defensores da teoria pragmadialética da escola de Amsterdã (VAN EEMEREN; GROOTENDORST, 1984), asseguram que uma teoria unificada geral da argumentação é necessária para subscrever qualquer coisa, exceto a pesquisa ad hoc em argumentação. Outros acreditam que trabalhos úteis sobre problemas específicos possam ser realizados independentemente do desenvolvimento de uma teoria global da argumentação. Embora aspectos da teoria pragmadialética tenham sido criticados (BLAIR; JOHNSON, 1993; WOODS, 1994; TINDALE, 1996), ela é uma das teorias mais abrangentes desenvolvidas até hoje. Se modificações são necessárias, quais são elas? A correta teoria global da argumentação poderia ser alguma versão da Pragmadialética? Caso contrário, qual seria a alternativa?

Quais tipos lógicos de argumento existem? A antiga distinção entre argumentos indutivos e dedutivos tem sido criticada por não ser exaustiva (ver "condutivo", "probatório" e "presumível” no item 5 acima).

Muitos dos itens das atuais pesquisas em curso listados acima se encaixam confortavelmente dentro da rubrica da "teoria da argumentação": os estudos históricos (item 1), as relações entre o diálogo e a natureza dialética da argumentação (item 2), a expansão do conceito de argumento (item 3), a relação entre razão e argumentação (item 4), o desenvolvimento de uma nova teoria da inferência (item 5), o estudo de esquemas argumentativos (item 6) e a elaboração de implicações teóricas de certos insights da teoria feminista (item 12).

2. A análise dos argumentos. Qual é a maneira correta de analisar argumentos específicos? Como o "correto" é aqui analisado? Parece evidente que a questão deva ser respondida em termos dos propósitos dessa análise; 
EID\&A - Revista Eletrônica de Estudos Integrados em Discurso e Argumentação, Ilhéus, n. 14, jul/dez.2017.

logo, eles precisam ser identificados. No fundo, trata-se de teorias sobre os possíveis tipos de argumento (o que nos traz de volta à teoria do argumento); um corolário é uma teoria sobre como os argumentos podem ser diagramados (ver item 7, acima). Os elementos retóricos (dentre outras possibilidades) e os lógicos devem ser registrados? Se sim, qual a melhor maneira de fazê-lo? A distinção retórica/lógica ainda permanece problemática, questão essa que volta à tona na análise argumentativa.

Adotar uma perspectiva mais ampla, no entanto, permite conceber a argumentação como um tipo de discurso. Consequentemente, as teorias da análise do discurso impactam a análise dos argumentos. Entramos aqui nos campos relacionados à pragmática.

Evidentemente, uma teoria geral da argumentação será pertinente para as questões relativas à análise de argumentos. Se os argumentos são essencialmente concebidos como diálogos entre indivíduos, isso exigirá um tipo de análise diferente do que se fossem entendidos como endereçados para o público em geral. Se os argumentos devem ser identificados como pertencentes a diferentes tipos esquemáticos, isso também afetará a forma como eles são analisados. Portanto, o item 2 (argumentação como diálogo), o item 3 (ampliação do conceito de diálogo), o item 5 (nova teoria da inferência), bem como o item 7 (estruturas e diagramas) encaixam-se todos na rubrica de análise de argumentos.

3. A teoria da avaliação. Aqui a questão motivadora inicial é: o que distingue um argumento "bom" de um "ruim"? A resposta será: depende da perspectiva. A narrativa padrão prevê que um argumento possa ser logicamente bom, retoricamente bom, ou mesmo bom a partir de uma série de outras perspectivas possíveis. No entanto, essas normas são independentes umas das outras? Alguns teóricos (como TINDALE, 1992, 1996, 1999; GILBERT, 1995) defendem que uma teoria da avaliação que integre diferentes perspectivas é necessária. As questões dos tipos de argumento e as normas relacionadas a eles também são aplicáveis aqui. Uma vez que a argumentação é entendida como um empreendimento social e funcional, a pragmática, a epistemologia social e a teoria da comunicação em geral (WILLARD, 1983, 1989) parecem ter impacto sobre sua avaliação. A pesquisa sobre as teorias da falácia e as falácias específicas (item 8 acima), bem como a análise detalhada dos critérios de uma boa argumentação (item 9 acima) também estão vinculadas a essa problemática. 
4. A teoria da crítica. Há muito que defendemos uma distinção entre a avaliação (identificação dos critérios do que seria bom ou ruim) e a crítica (o ato da crítica, incluindo não só a aplicação dos critérios de uma boa ou má argumentação, mas também o ato público de crítica). A crítica argumentativa pressupõe uma teoria normativa da função dessa crítica. Tal teoria, por sua vez, pressupõe uma teoria normativa dos papéis da argumentação. Se o argumento é corretamente utilizado para alcançar um conjunto de objetivos, então a crítica pública da argumentação deve estar relacionada a sua contribuição para atingir tais objetivos (ver WALTON, 1990). É no âmbito desta problemática que situamos o item 10 (desenvolvimentos na teoria da crítica argumentativa), o item 11 (investigação do papel social da argumentação) e o item 12 (impacto da teoria feminista).

\section{Lógica informal: Recursos}

1. Periódicos. A Informal Logic tem sido o periódico de registro da área desde 1983, mas artigos sobre tópicos relacionados à lógica informal também aparecem regularmente em Argumentation (fundada em 1986), Philosophy and Rhetoric, Argumentation and Advocacy (o periódico da American Forensic Association) e Inquiry: Critical Thinking Across the Disciplines (fundada em 1988). Artigos sobre tópicos de lógica informal também apareceram em uma ampla gama de periódicos filosóficos gerais, incluindo, mas não se restringindo a, as revistas Synthese, Logique et Analyze e a American Philosophical Quarterly.

2. Programas acadêmicos. Até onde sabemos, não há programas de graduação ou pós-graduação dedicados à lógica informal. Praticamente todas as faculdades e universidades nos Estados Unidos e no Canadá têm um curso de nível introdutório que trata da lógica informal em alguma medida (geralmente como parte de uma orientação sobre "pensamento crítico" ou "raciocínio", ou ainda uma introdução ao curso de lógica). Conhecemos apenas um pequeno conjunto de cursos de graduação e alguns outros cursos de Mestrado ou Doutorado, principalmente no Canadá, dedicados à área.

3. Congressos. Os congressos iniciais sobre lógica informal aconteceram na University of Windsor (Windsor, Ontário) em 1978, 1983 e 1989. Congressos sobre a lógica informal e o pensamento crítico têm sido ${ }^{3}$ realizados na

\footnotetext{
${ }^{3}$ N.T. O autor se refere ao período compreendido entre 1981 e a data da publicação original (2000).
} 
EID\&A - Revista Eletrônica de Estudos Integrados em Discurso e Argumentação, Ilhéus, n. 14, jul/dez.2017.

Sonoma State University (Rohnert Park, California) anualmente, desde maio de 1981; na Christopher Newport University (Newport News, Virgínia), ocorreram todos os anos entre 1984 e 1988. A Oakton Community College recebeu cinco congressos sobre reflexão crítica de 1988 a 1992 (Des Plains, Illinois) e outro evento foi realizado na George Mason University (Fairfax, Virginia) em 1995. A Association for Informal Logic and Critical Thinking, desde a sua criação em 1983, organizou sessões sobre lógica informal em conjunto com as reuniões das divisões oriental, central e pacífica da American Philosophical Association e da Canadian Philosophical Association. Publicações sobre lógica informal estiveram no programa dos congressos da International Society for the Study of Argumentation de 1986, 1990, 1994 e 1998 (University of Amsterdam, The Netherlands). Finalmente, a Ontario Society for the Study of Argumentation realizou dois eventos dedicados, em grande parte, à lógica informal em 1995, 1997 e 1999 (Brock University, Ontário).

4. Desafios. Em nossa opinião, o campo da lógica informal enfrenta dois principais desafios práticos, os quais estão relacionados entre si. É necessário encontrar apoio e recursos para a formação em termos de pós-graduação para que jovens estudiosos tomem consciência de sua problemática e literatura, de forma a fazer da lógica informal uma área de concentração em seus programas de pesquisa. Além disso, ela precisa adentrar o campo filosófico, de modo que seus achados teóricos se tornem conhecidos e mais bem refletidos nos cursos de graduação.

\section{Implicações para a Filosofia}

Que implicações a lógica informal tem para a filosofia, e, de fato, para o mundo em geral que habitamos?

1. O fim do dedutivismo. O raciocínio e a argumentação filosóficos precisam da análise lógico-informal. É um preconceito teórico, e não um fato, assumir que o único raciocínio e a única argumentação filosófica respeitável seja a que emprega inferências dedutivas. Ryle (1954, p. 112) reconhece isso quando afirma:

Se um dado argumento filosófico é válido ou falacioso, é, em geral, uma questão debatível em si. Uma inspeção simples não pode decidir. Mais frequentemente, é uma questão de o argumento ter muita, pouca ou nenhuma força. 
Talvez a contribuição mais importante da lógica informal seja auxiliar a concluir a revolução iniciada pelos pragmaticistas que se voltaram à teoria clássica (platônica/cartesiana) do conhecimento. O trabalho deles pode ser visto como uma tentativa de reconceptualizar o conhecimento de acordo com o modelo das ciências empíricas. O trabalho em lógica informal pode ser entendido como uma tentativa de reconceptualizar a argumentação e libertála de suas ligações históricas com o que Toulmin e Perelman denominaram modelo geométrico ou matemático. Isso significa, entre outras coisas, o fim do dedutivismo - a ideia de que todas as implicações são ou dedutivas ou defectivas; o fim da noção de que o argumento deveria ser compreendido como prova; e o fim de uma divisão de classes entre tipos de crenças - crenças de elite ("de primeira") seriam verdades necessárias ou derivadas de premissas tidas como verdadeiras, enquanto crenças "de segunda" seriam as garantidas por algum cálculo de probabilidade, e todas as demais seriam intocáveis, não viabilizando aceitação por uma pessoa razoável.

2. O desaparecimento da visão de que a lógica é a teoria do raciocínio. Ao identificar raciocínio com implicação e considerar que a lógica é essencialmente dedutiva, foi possível acreditar que a lógica fosse, de fato, idêntica à teoria do raciocínio. Entretanto, uma vez adotada uma visão mais ampla de lógica e de raciocínio, torna-se claro que há mais questões envolvidas na construção de uma teoria do raciocínio do que a lógica dedutiva formal pode abarcar (FINOCCHIARO, 1984; JOHNSON, 1996). Se estivermos certos, a educação filosófica precisa alterar sua narrativa padrão sobre argumentação, raciocínio e lógica.

Outra implicação do trabalho em lógica informal tem sido deixar claro que a lógica ainda está em desenvolvimento. Ela não carrega essa responsabilidade sozinha, contudo; outros desenvolvimentos também assim atestam, como a lógica difusa (ZADEH, 1975) e a lógica dinâmica (VAN BENTHEM, 1995). Entretanto, como já mencionado, o vasto campo de educação filosófica permanece intocado pelos achados de lógica informal. Como consequência, uma séria deseducação continua.

3. A reavaliação do formalismo. Outro resultado da lógica informal tem sido desafiar a forte ligação ao formalismo e tudo o que a ele se relaciona: algoritmos, procedimentos de prova, teorias modelares, etc. Assim, não é apenas o viés dedutivo inerente à lógica tradicional que a lógica informal tem 
EID\&A - Revista Eletrônica de Estudos Integrados em Discurso e Argumentação, Ilhéus, n. 14, jul/dez.2017.

ajudado a revelar, mas também a preferência pelo formalismo. É a isso que Toulmin (1992, p. 4) se refere quando afirma:

A partir de meados do século XVII, os Filósofos Modernos consideraram os problemas formais como centrais - sobretudo porque seriam discutidos em termos gerais, "descontextualizados". Assim, lógica se tornou o equivalente a lógica formal.

Aqui, é crucial enfatizar a discussão realizada anteriormente de que há diferentes sentidos do termo 'formal' (ver BARTH; KRABBE, 1982; JOHNSON; BLAIR, 1990). Essa constatação abre espaço para compreender que lógica informal não é, portanto, uma contradição ou um oxímoro, como alguns têm alegado. Nós desejamos enfatizar que lógica informal não é de modo algum incompatível com procedimentos, aplicação de critérios ou rigor. Trata-se da questão de quais critérios são usados, e, aqui, a lógica informal é informal porque rejeita, por um lado, a visão logicista de que lógica formal (ao estilo de Russell) possui a chave para o entendimento da estrutura de todos os argumentos, e, por outro, a visão de que a validade é o padrão apropriado a ser exigido de todo argumento.

Um outro modo de asseverar esta questão é dizer que a lógica informal está aliada ao movimento de tornar a lógica mais empírica e menos a priorística (BARTH, 1992; TOULMIN, 1958; WEINSTEIN, 1990).

\section{Implicações práticas mais amplas}

Finalmente, podemos alegar que a prática de argumentação tem passado por tempos difíceis na cultura ocidental. Observadores sérios têm notado um decréscimo nas habilidades de letramento, o debate público parece ter alcançado o pior nível de todos os tempos, e a retórica pública é dominada pelo modo confessional de "talk-shows" televisivos. Onde, em meio a tudo isso, a prática da argumentação deveria ser apreciada e nutrida senão na Academia? O ensino de padrões elevados de interpretação, avaliação e crítica de argumentos que têm aplicações práticas é o objetivo do lado pedagógico da lógica informal.

Fora da Academia, no que alguns chamariam de "mundo da vida" ("lifeworld"), nós testemunhamos o desaparecimento da antiga ordem mundial. As coalizões pós-Segunda Guerra Mundial existentes sob a ameaça da força e do poder militar estão, agora, em todos os lugares abrindo espaço para novos 
EID\&A - Revista Eletrônica de Estudos Integrados em Discurso e Argumentação, Ilhéus, n. 14, jul/dez.2017.

alinhamentos baseados em interesses comuns e persuasão racional. Os estados Balcânicos, o Oriente Médico, partes da África e o subcontinente Índia-Paquistão são notáveis exceções. A comunidade humana deve entender que a única força da qual podemos nos valer é "a força do melhor argumento". Entretanto, paradoxalmente, parece que precisamente quando há uma grande necessidade por argumentação no mundo da vida, esta nunca esteve em tamanho perigo como prática cultural nas próprias sociedades baseadas em sua operação saudável, as sociedades democráticas. Mais do que nunca, nós, a comunidade filosófica, e, particularmente, aqueles comprometidos com o estudo da argumentação cotidiana, temos algo a contribuir na educação do mundo.

Por meio do compromisso com o desenvolvimento de melhores teorias de argumentação, a lógica informal tem um papel importante não apenas para a academia e para uma teoria do raciocínio, mas também para o mundo da vida.

\section{Referências}

AYIM, M. Violence and domination as metaphors in academic discourse. In: GOVIER, T. (Ed.). Selected issues in logic and communication. Belmont, CA: Wadsworth, 1988.

BARRY, V.; RUDNOW, J. Invitation to critical thinking. 2 ed. New York: Thomson Wadswoth, 1992.

BARTH, E. A new field: empirical logic/bioprograms, logemes and logics as institutions. Synthese. Dordrecht, v. 63, p. 376-388, 1985.

; KRABBE, E. C. W. From axiom to dialogue. A philosophical study of logics and argumentation. Berlin/New York: Walter de Gruyter, 1982.

BENTHEM, J. van. Logic and argumentation. In: EEMEREN, F. H. van; GROOTENDORST, R.; BLAIR, J. A.; WILLARD, C. A. (Eds.). Proceedings of the third international conference on argumentation. Amsterdã, v. 1, 1995, p.18-35.

BIRDSELL, D. S.; GROARKE, L. Toward a theory of visual argument. Argumentation and Advocacy. Chicago, v. 33, n.1, p. 1-10, 1996.

BLAIR, J. A. What is the right amount of support for a conclusion? In: EEMEREN, F. H. van; GROOTENDORST, R.; BLAIR, J. A.; WILLARD, C. A. (Eds.). Proceedings of the second international conference on argumentation. Amsterdam, v. 1, 1991, p. 330-337. 
EID\&A - Revista Eletrônica de Estudos Integrados em Discurso e Argumentação, Ilhéus, n. 14, jul/dez.2017.

. Premise adequacy. In: EEMEREN, F. H. van; GROOTENDORST, R.; BLAIR, J. A.; WILLARD, C. A. (Eds.). Perspectives and approaches. Proceedings of the third ISSA conference on argumentation. Amsterdã, v.2, 1995, p. 191-202.

- The possibility and actuality of visual arguments. Argumentation and Advocacy. Chicago, v.33, p.23-39, 1996.

. Premissary relevance. Argumentation. Dordrecht, v.6, p.203-217, 1992.

- Walton's argument schemes for presumptive reasoning: $A$ critique and development. In: EEMEREN, F. H. van; GROOTENDORST, R.; BLAIR, J. A.; WILLARD, C. A. (Eds.). Proceedings of the fourth International Society for the Study of Argumentation conference. Amsterdam: SicSat, 1999, p. 56-61.

; JOHNSON, R. H. The current state of informal logic and critical thinking. Informal Logic, Windsor, v. 9, p. 147-151, 1987.

.; JOHNSON, R. H. Dissent in fallacyland. Part I: Problems with Van Eemeren and Grootendorst. In: MCKERROW, R. E. (ed.). Proceedings of the Eighth SCA/AFA Conference on Argumentation. Annandale, VA: Speech Communication Association, 1993, p. 1880190.

CARNEY, J. D.; SHEER, R. K. Fundamentals of logic. New York: Macmillan, 1964.

COPI, I. Introduction to logic. 7 ed. New York: Macmillan, 1986.

. Informal logic. New York: Macmillan, 1986.

DAMER, T. E. Attacking faulty reasoning. 2 ed. Belmont, CA: Wadsworth, 1987.

FINOCCHIARO, M. Informal logic and the theory of reasoning. Informal Logic, Windsor, v. 6, n. 2, p. 3-8, 1984.

. Methodological problems in empirical logic. Communication and Cognition. v. 22, p. 313-335, 1989 .

FISHER, A.; SCRIVEN, M. Critical thinking: It's definition and assessment. Point Reyes, CA: Edgepress, 1997.

FREEMAN, J. B. Thinking logically: Basic concepts for reasoning. Englewood Cliffs, NJ: Prentice-Hall, 1988.

- Dialectics and the macrostructure of argument: A theory of argument structure. Amsterdam: Mouton de Gruyter. Waveland Press, 1991.

- Relevance, Warrants, Backing, Inductive Support. Argumentation. Dordrecht, v .6, 1992, p.219-235. 
EID\&A - Revista Eletrônica de Estudos Integrados em Discurso e Argumentação, Ilhéus, n. 14, jul/dez.2017.

. Premise acceptability, deontology, internalism, justification. Informal Logic, Windsor, v. 17, n. 2, p. 270-278, 1995.

GILBERT, M. A. Feminism, argumentation and coalescence. Informal Logic, Windsor, v. 16, n 2, p. 95-113, 1995.

. Coalescent argumentation. Mahwah, NJ: Lawrence Erlbaum Associates, 1997.

GOLDMAN, A. Epistemology and cognition. Cambridge: MIT Press, 1986.

GOVIER, T. Problems in argument analysis and evaluation. Dordrecht: Foris Publications, 1987.

. When logic meets politics: Testimony, distrust and rhetorical disadvantage. Informal Logic, Windsor, v. 15, n.2, p. 93-104, 1993.

GROARKE, L. Logic, art and argument. Informal Logic, Windsor, v. 18.2, 1996, p. 105-129.

HAMBLIN, C. L. Fallacies. Londres: Methuen, 1970.

HANSEN, H. V. An informal logic bibliography. Informal Logic, Windsor, v. 12, p. 155184, 1990.

; PINTO, R. C. (Eds.). Fallacies: Classical and contemporary readings. University Park, PA: The Pennsylvania State University Press, 1995.

HINTIKKA, J. The fallacy of fallacies. Argumentation, Dodrecht, v. 1, 211-238, 1987.

HITCHCOCK, D. Relevance. Argumentation. Dordrecht, v.6, p. 251-270, 1992.

JOHNSON, R.H. The rise of informal logic. Newport News, VA: Vale Press, 1996.

- Manifest rationality: A pragmatic theory of argument. Mahwah, NJ: Lawrence Erlbaum Associates, 2000.

U.S. ed., 1994.

; BLAIR, J. A. Logical self-defense. [1977]. 3 ed., Toronto: McGraw Hill Ryerson.

; BLAIR, J. A. Logical self-defense. [1977]. New York: McGraw-Hill, 1993.

; BLAIR, J. A. Contexts of informal reasoning: Commentary. In: VOSS, J.; PERKINS, D.; SEGAL, J. W. (Eds.). Informal Reasoning and Education. Hillsdale, NJ: Lawrence Erlbaum Associates, 1991, p.131-150.

KAHANE, H. Logic and contemporary rhetoric. Belmont, CA: Wadsworth, 1971.

KIENPOINTNER, M. Alltagslogik: struktur und funktion von argumentationsmustern. Stuttgart: frommann-holzboog, 1992. 
LITTLE, J. F.; GROARKE, L. A.; TINDALE, C. W. Good reasoning matters. Toronto: McLelland and Stewart, 1989.

MCPECK, J. Critical thinking and education. New York: St. Martin's Press, 1981.

MENSSEN, S. Do women and men use different logics? A reply to Carol Gilligan and Deborah Orr. Informal Logic, Windsor, v. 15, n.2, p. 123-138, 1993.

NYE, A. Words of power. New York: Routledge, 1990.

ORR, D. Just the facts Ma'am: Informal logic, gender and pedagogy. Informal Logic, Windsor, v. 11, n. 1, p. 1-10, 1989.

. On logic and moral voice. Informal Logic, Windsor, v. 17, n. 3, p. 347-363, 1995.

PINTO, R. C.; BLAIR, J. A. Reasoning: a practical guide. Englewood Cliffs, NJ: PrenticeHall, 1993.

PINTO, R. C. The relation of argument to inference. In: VAN EEMEREN, F. H.; GROOTENDORST, R.; BLAIR, J. A.; WILLARD, C. A. (Eds.). Perspectives and approaches: Proceedings of the Third ISSA Conference on Argumentation, Amsterdam, June 1994. Amsterdam: SICSAT, 1995, p. 271-286.

RESCHER, N. Plausible reasoning: an introduction to the theory and practice of plausible inference. Assen: Van Gorcum, 1976.

. Dialectics: A Controversy-oriented approach to the theory of knowledge. Albany: State University of New York, 1977.

RYLE, G. Dilemmas. Cambridge: Cambridge University Press, 1954.

SCRIVEN, M. Probative logic: Review and preview. In: VAN EEMEREN, F. H.; GROOTENDORST, R.; BLAIR, J. A.; WILLARD, C. A. (Eds.). Argumentation across the lines of discipline. Dordrecht/Providence: Foris Publications, p.7-32, 1987.

SEECH, Z. Logic in everyday life: Practical reasoning skills. Belmont: Wadsworth, 1992.

SIEGEL, H. Educating reason: Rationality, critical thinking and education. New York: Routledge, 1988.

SNOECK HENKEMANS, A. F. Analysing complex argumentation. Amsterdam: SicSat, 1992.

TINDALE, C. W. Audiences, relevance and cognitive environments. Argumentation, Springer, v. 6, p. 177-188, 1992. 
EID\&A - Revista Eletrônica de Estudos Integrados em Discurso e Argumentação, Ilhéus, n. 14, jul/dez.2017.

. Fallacies in transition: An assessment of the pragma-dialectical perspective. Informal Logic, Windsor, v. 18, n. 1, p. 17-33, 1996.

. Acts of arguing: A rhetorical model of argument. Albany: State University of New York Press, 1999.

TOULMIN, S. E. The uses of argument. Cambridge: Cambridge University Press, 1958.

. Logic, rhetoric and reason: redressing the balance. In: VAN EEMEREN, F. H.; GROOTENDORST, R.; BLAIR, J.A.; WILLARD, C. A. (Eds.). Argumentation illuminated. Amsterdam: SicSat, 1992, p. 3-11.

VAN EEMEREN, F. H.; GROOTENDORST, R. Speech acts in argumentative discussions. Dordrecht/Providence: Foris Publications, 1984.

; . Argumentation, communication and fallacies: A pragma-dialectical perespective. Hillsdale, NJ: Lawrence Erlbaum Associates, 1992.

WALTON, D. Informal fallacies: Towards a theory of argument criticisms. Amsterdam: John Benjamins, 1987.

.Informal logic: A handbook for critical argumentation. Cambridge: Cambridge University Press, 1989.

. Begging the question. Westport, CT: Greenwood, 1991.

. The place of emotion in argument. University Park, PA: The Pennsylvania State University Press, 1992a.

. Slippery slope arguments. Oxford: Oxford University Press, 1992b.

. Arguments from ignorance. University Park, PA: The Pennsylvania State University Press, 1994.

. A pragmatic theory of fallacy. Tuscaloosa/London: The University of Alabama Press, 1995a.

- Argument structure: A pragmatic theory. Toronto: University of Toronto Press, 1996a.

. Argumentation schemes for presumptive reasoning. Mahwah, $\mathrm{NJ}$ : Lawrence Erlbaum Associates, 1996b.

. Ad hominem arguments. Tuscaloosa: University of Alabama Press, 1998.

; BRINTON, A. (Eds.). Historical foundations of informal logic. Aldershot: Ashgate, 1997. 
EID\&A - Revista Eletrônica de Estudos Integrados em Discurso e Argumentação, Ilhéus, n. 14, jul/dez.2017.

WEINSTEIN, M. Towards a research agenda for informal logic and critical thinking. Informal Logic, Windsor, v. 12, p. 121-143, 1990.

WALTON, D. Informal logic and applied epistemology. In: JOHNSON, R. H.; BLAIR, J. A. (Eds.). New essays in informal logic. Windsor: Informal Logic, 1994.

WELLMAN, C. Challenge and response, justification in ethics. Carbondale e Edwardsville, IL: Southern Illinois University Press. London/Amsterdam: Feffer \& Simons, Inc., 1971.

WILLARD, C. A. Argumentation and the social grounds of knowledge. Tuscaloosa: The University of Alabama Press, 1983.

. A theory of argumentation. Tuscaloosa: The University of Alabama Press, 1989.

WOODS, J. Apocalyptic relevance. Argumentation. Dordrecht, v. 6, 1992, p.189-202.

. Is the theoretical unity of the fallacies possible? Informal Logic, Windsor, v. 16, n. 2, p.77-85, 1994.

WOODS, J. Fearful symmetry. In: HANSEN, H. V.; PINTO, R. C. (Eds.). Fallacies: Classical and contemporary readings. University Park, PA: The Pennsylvania State University Press, 1995.

Ryerson, 1982.

; WALTON, D. Argument, the logic of the fallacies. Toronto: McGrawHill .; WALTON, D. Fallacies: Selected papers, 1972-1982. Dordrechtl Providence, Foris Publications, 1989.

ZADEH, L. Fuzzy logic and approximate reasoning. Synthese. Dordrecht, v.30, 1975, p.407-428.

Tradução:

Paulo Roberto Gonçalves-Segundo

Docente pelo Programa de Pós-Graduação em Filologia e Língua Portuguesa da Universidade de São Paulo (USP)

E-mail: paulosegundo@usp.br

Danielle Martins Santos

Graduanda em Língua Portuguesa e Inglês pela Universidade de São Paulo (USP)

E-mail: danielle.martins.santos@usp.br 
EID\&A - Revista Eletrônica de Estudos Integrados em Discurso e Argumentação, Ilhéus, n. 14, jul/dez.2017.

Gabriel Isola Lanzoni

Mestrando pelo Programa de Pós-Graduação em Filologia e Língua Portuguesa da Universidade de São Paulo (USP)

E-mail: gabriel.lanzoni@usp.br

Maria Gabriela Rodrigues de Castro

Mestranda pelo Programa de Pós-Graduação em Filologia e Língua Portuguesa da Universidade de São Paulo (USP)

E-mail: maria.gabriela.castro@alumni.usp.br

Winola Weiss Pires Cunha

Mestranda pelo Programa de Pós-Graduação em Filologia e Língua Portuguesa da Universidade de São Paulo (USP)

E-mail:winola.weiss@usp.br

Forma de citação sugerida:

JOHNSON, Ralph H.; BLAIR, J. Anthony. Lógica informal: uma visão geral. Trad. Paulo Roberto Gonçalves-Segundo et al. EID\&A - Revista Eletrônica de Estudos Integrados em Discurso e Argumentação, Ilhéus, n. 14, p. 195-215, jul/dez.2017.

Recebido em: 31/08/2017

Aprovado em: 06/09/2017 